\title{
Effect of Conduction Pre-heating in Au-Al Thermosonic Wire Bonding
}

\author{
Gurbinder Singh*, Othman Mamat \\ Department of Mechanical Engineering, Universiti Teknologi Petronas, Malaysia Bandar Seri Iskandar, Tronoh, Perak, Malaysia. \\ Email: "gurbinder_g01395@utp.edu.my
}

Received August 20 ${ }^{\text {th }}, 2011$; revised September 24 ${ }^{\text {th }}$, 2011; accepted October $3^{\text {rd }}, 2011$.

\begin{abstract}
This paper presents the recent study by investigating the vital responses of wire bonding with the application of conduction pre-heating. It is observed through literature reviews that, the effect of pre-heating has not been completely explored to enable the successful application of pre-heating during wire bonding. The aim of wire bonding is to form quality and reliable solid-state bonds to interconnect metals such as gold wires to metalized pads deposited on silicon integrated circuits. Typically, there are 3 main wire bonding techniques applied in the industry; Thermo-compression, Ultrasonic and Thermosonic. This experiment utilizes the most common and widely used platform which is thermosonic bonding. This technique is explored with the application of conduction pre-heating along with heat on the bonding site, ultrasonic energy and force on an Au-Al system. Sixteen groups of bonding conditions which include eight hundred data points of shear strength at various temperature settings were compared to establish the relationship between bonding strength and the application of conduction pre-heating. The results of this study will clearly indicate the effects of applied conduction pre-heating towards bonding strength which may further produce a robust wire bonding system.
\end{abstract}

Keywords: Conduction Pre-Heating, Intermetallic Coverage, Shear Strength, Thermosonic Wire Bonding

\section{Introduction}

The revolution that began over a hundred years ago with the discovery of electricity and the rapid electrification of your society has transformed the means of communication amongst many others. The electronics industry today is driving for speedier data processing and more efficient data acquisition and transmission. With these requirements, means and methods of creating a robust and reliable bonding within an electronic chip is gaining importance.

From the three typical, wire bonding techniques mentioned, thermosonic wire bonding has been prevalent in the application of solid state inter-connect technology. This bonding method may also be known as diffusion bonding. In the process of making an interconnection, two wire bonds are formed. The first bond involves the formation of a ball with electric flame off (EFO) process.

Factors such as ultrasonic energy, temperature, and pressure may influence the quality of bonding quality $[1,2]$. In addition, diffusion bonding processes depend for their success on a combination of three factors. The first being the absence of contamination at the mating surfaces; secondly, the ability of at least one component to undergo sufficient plastic flow in order to develop complete contact across the interface between the two joining metals and lastly, sufficient time for diffusion to occur in the interface region in order to eliminate micro-structural instability and establish adequate bonding strength [3].

Upon application of the primary factors to form an intermetallic coverage (IMC) that makes the connection on the bond pad of a die, the wire is then lifted to form a loop and placed in contact with the desired bond area of a leadframe to form a wedge bond. In this process, the bonding temperature is one of the main bonding parameters which play an important role in the bonding [4]. Essentially, different temperature will lead to different bonding output response as different temperature conditions mean different bonding environment. In previous studies, a parabolic relationship between temperature and strength has been determined. Too low or too high temperature during bonding can lead to unsuccessful bonding or low bonding strength $[5,6]$.

Although many studies about temperature effect in wire bonding technology has been carried out, however research findings on conduction pre-heating for the application on wire bonding is nonexistent. Therefore it is worth investigating the effects of conduction pre-heat 
application on thermosonic wire bonding while keeping other factors at constant. The results of this study will clearly indicate the effects of applied pre-heat towards bonding strength which may further produce a robust wire bonding system. Sixteen groups of bonding conditions which includes eight hundred data points of shear strength at various temperature settings were compared to establish the relationship between bonding strength and the application of conduction pre-heating.

\section{Experimental Details}

This study was carried out using a $138 \mathrm{KHz}$ Thermosonic wire bonder. The bonding temperature was tuned to produce different thermosonic bonding process while all other bonding parameters such as bonding ultrasonic power, bonding force and time were as constant. The bonding parameters are listed in Table 1.

The diameter of gold wire (99.99\% Au) used is $1 \mathrm{mil}$. The gold wire bonding was performed on a $16 \mathrm{mil} \times 16$ mil die size with 1um thickness metal composition $\mathrm{Al}-99.5 \%$, Cu- $0.5 \%$. The bond pad opening is $4 \mathrm{mil} \times 4$ mils and bond pad pitch is 5 mils. The following is an outline of the gold wire bonding procedure which was carried out. Refer to Figure 1.

(i) The Au wire at the capillary tip was melted to produce the Free air ball (FAB) using discharge from electric flame off (EFO)

(ii) The capillary is lowered and the free air ball is compressed onto the $\mathrm{Al}$ pad for bonding.

(iii) The capillary is lifted and the Au wire is connected to the lead to produce a wedge bond.

(iv) The Au wire is cut. (repeat step 1-4)

The bonding temperature was measured by a K-type

Table 1. Bonding Parameters.

US Power Bond Force (gm) Bond Time (ms) Temperature $\left({ }^{\circ} \mathrm{C}\right)$

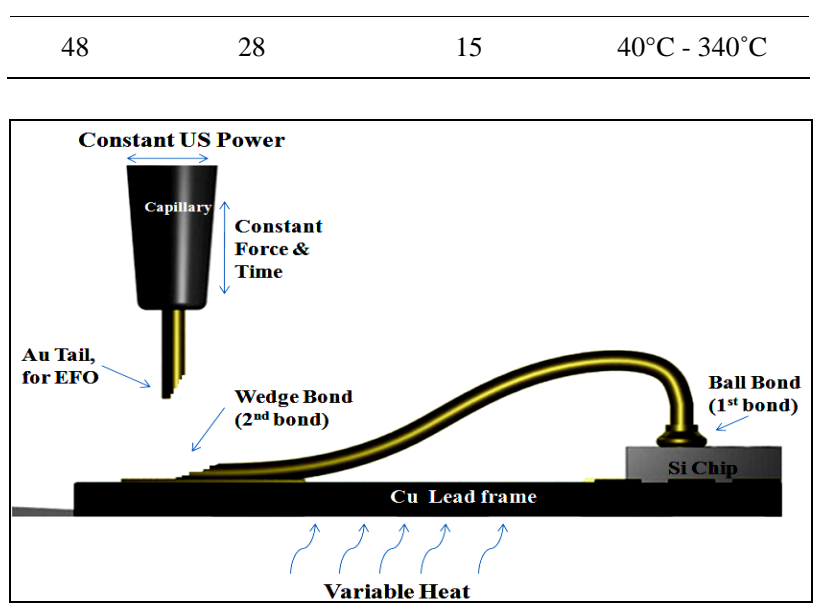

Figure 1. Thermosonic wire bonding setup. thermocouple sensor with a measurement range of $0^{\circ} \mathrm{C}$ $500^{\circ} \mathrm{C}$. A total of 16 runs were performed with bonding temperature range of $40^{\circ} \mathrm{C}$ to $340^{\circ} \mathrm{C}$ with a step of $20^{\circ} \mathrm{C}$. The bonding experiments were consistently repeated for 50 times under each testing condition with heater block without conduction pre-heating and with conduction pre-heating for statistical analysis.

Upon completing the samples, the output response of the thermosonic bonding with and without conduction pre-heating were assessed using Royce 550 wire bond tester. The destructive shear strength between the gold ball and substrate is a common judgment for bondability $[7,8]$.

To further analyze the bondability, intermetallic coverage assessment was also carried out using fuming potassium hydroxide, $\mathrm{KOH}$. Cross sectioning using grinding and polishing machines were used to enable analysis on gold ball bond. Samples were observed using an optical microscope and scanning electron microscope (SEM). Thickness and diameter of the ball bond profile and Intermetallic growth was measured using micrometer scale attached to the optical microscope and the scope was calibrated using the standards provided with the scope.

\section{Results and Discussion}

\subsection{Effect of Conduction Pre-heating on Bonding Strength in Au-Al Wire Bonding System}

The result of this study showed, there was significant difference in the bonding strength response with the usage of conduction pre-heating as compared against the conventional non conduction pre-heated samples. This can be seen from the one-way analysis of ball shear strength by heater block design, as shown in Figure 2.

The effect of heat on wire bonding was measurable not only against ball shear strength but also by the representation

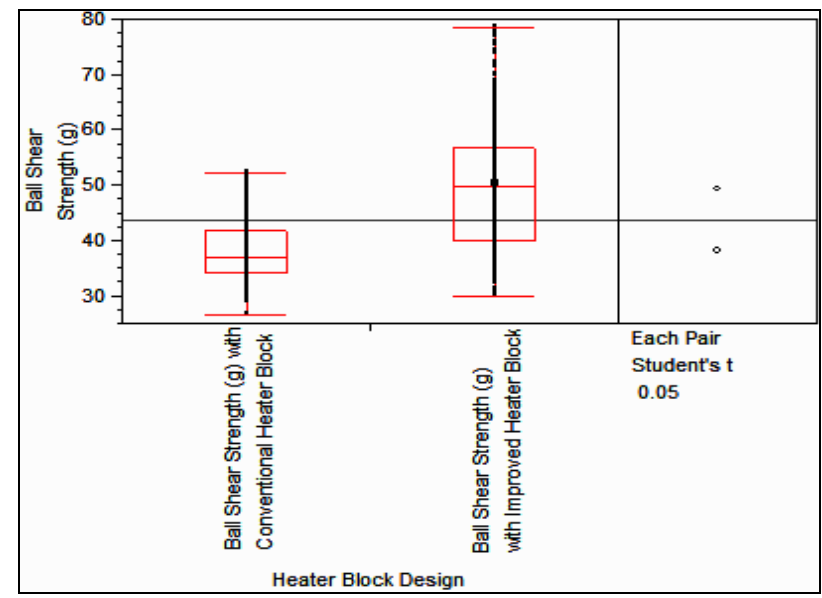

Figure 2. Ball shear strength by heater block design 
of intermetallic coverage. IMC which is denoted by the darker colored area is the result of diffusion in between $\mathrm{Au}$ and $\mathrm{Al}$. The metallic properties contained in this area consist of AuAl.

It was observed that, by using the conventional non conduction pre-heat block, too low or too high temperature may result in unfavorable intermetallic coverage. However, by using the conduction pre-heat block, it was able to produce homogeneous intermetallic coverage at all assessed levels. This observable fact can be further explained by the following related theories [9-11]

a. The presence of oxide and other forms of contamination may not be able to be removed with inadequate heat and may impede diffusion between the bonding metals. This may lead to unsuccessful bonding or lower bonding strength with poor IMC.

b. The application of appropriate and adequate temperature will soften the metal surface and accelerate the diffusion between the bonding systems.

As such, it was evident that, adequate heat supplied via conduction pre-heating is able to deliver improved intermetallic coverage. This can be seen by the results of intermetallic coverage provided in Table 2.

\subsection{Effect of Conduction Pre-heating on IMC Voiding in Au-Al Wire Bonding System}

Results of this study showed significant difference in the formation of voids with respect to different temperature levels applied with and without conduction pre-heating. From the study, we are able to understand that the characteristics of ball bond are able to be altered physically. Research carried out previously with the application of short-time Fourier transform (STFT) to input/ output power of an ultrasonic transducer is able to deduce this phenomena [12,13].

The cross sectioning image in Figure 3 shows the difference in IMC growth with the application on non conduction pre-heat heater block and with conduction pre-heat heater block. At $180^{\circ} \mathrm{C}$ the IMC growth seems to be thicker (3um) with the application of conduction pre-heat. With the application of pre-heat, the IMC formation is homogeneous and there are no voids observed.

Results of study at temperature $220^{\circ} \mathrm{C}$ in Figure 4 shows; the IMC thickness has grown to 3.1um and 3.4 respectively with the usage of non conduction pre-heat and pre-heat heater block. The IMC growth seen at this stage shows obvious voiding with the application of non pre-heat heater block. The IMC formation with the usage of pre-heat heater block remains to be homogeneous and without voids.

The result of this study clearly shows, the application
Table 2. Comparison of IMC
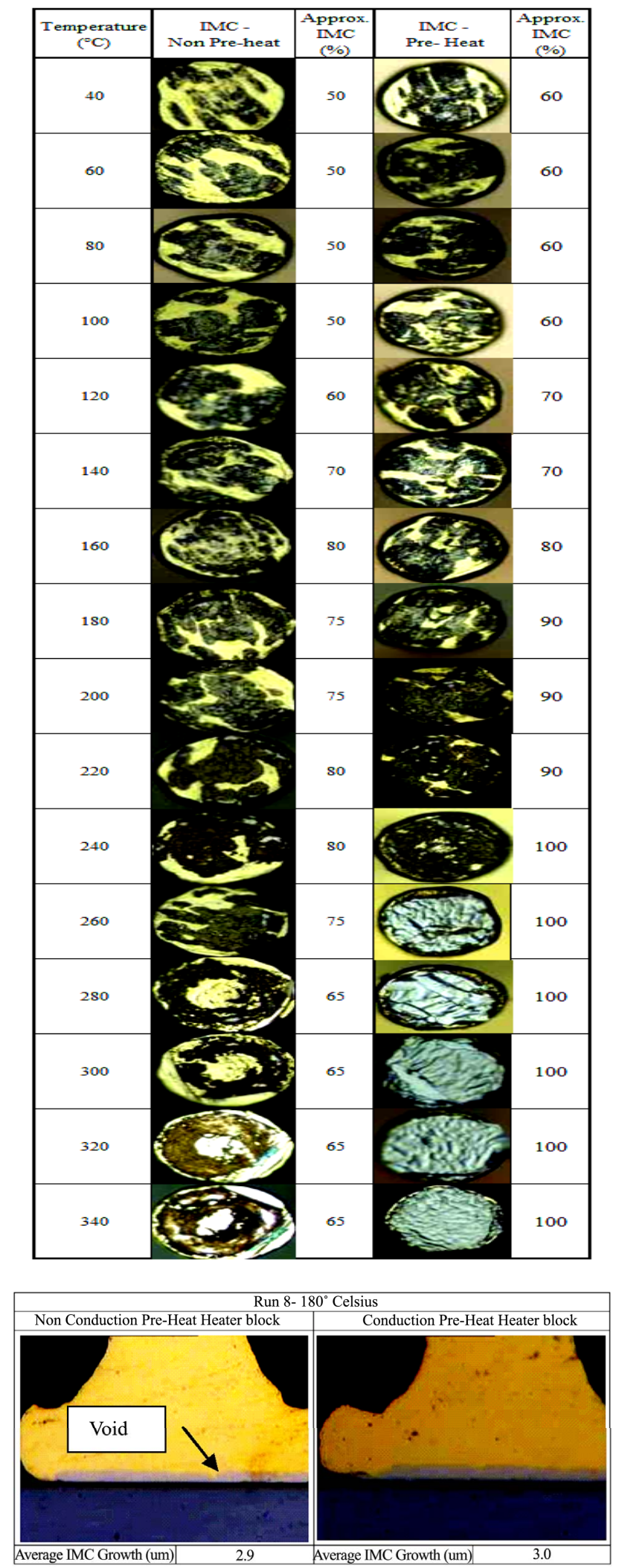

Figure 3. IMC growth at $180^{\circ} \mathrm{C}$. 


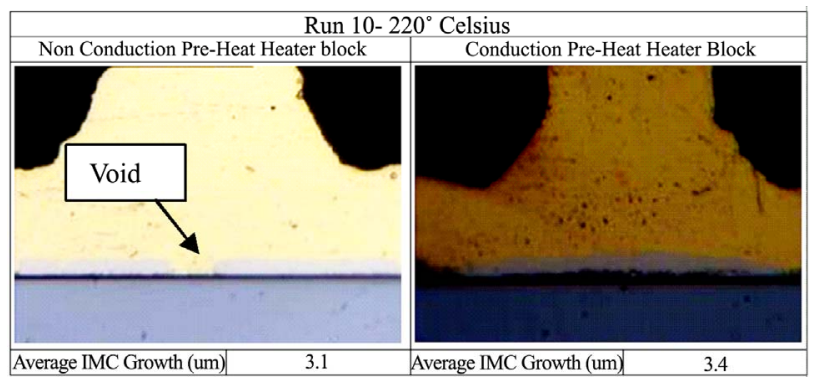

Figure 4. IMC growth at $220^{\circ} \mathrm{C}$.

of conduction pre-heat does have positive impact towards improved IMC formation. Previous theories (Long et al., 2008) have concluded, with the usage of adequate heating environment, the impedance of piezo-transducer is high and the consumed power of piezo-transducer is low. This effect may exert adequate physical bonding power to the bonded aluminum interface and result in IMC formation which is homogeneous and without voids.

\section{Conclusions}

From the study and measurements carried out, the effect of conduction pre-heating in $\mathrm{Au}-\mathrm{Al}$ thermosonic wire bonding was investigated. It is clearly revealed that, adequate heat supplied via conduction pre-heating is able to deliver improved intermetallic coverage. This was evident in the significantly increased ball shear strength values and the improved intermetallic formation. In addition, high optical magnification on the cross sectioned samples also showed an improvement in the area of void formation which is critical in ensuring good bondability.

It is concluded, the application of conduction preheating unaccompanied by other bonding factors, has an effect in Au-Al thermosonic wire bonding. The objective of this study has been achieved. In future, further in depth studies to assess the reliability of parts with conduction pre-heating on bonding surfaces should be explored for enhanced wire bondability.

\section{REFERENCES}

[1] J. Antony, "Improving the Wire Bonding Process Quality Using Statistically Designed Experiment,” Journal of Microelectronics, Vol. 30, No. 2, 1999, pp. 161-168. doi:10.1016/S0026-2692(98)00104-9

[2] Z. N. Liang and F. G Kuper, “A Concept to Relate Wire
Bonding Parameters to Bondability and Ball Bond Reliability,” Microelectronics Reliability, Vol. 38, No. 6-8, 1998, pp. 1278-1291. doi:10.1016/S0026 2714(98)00093-6

[3] D. Brandon and W. Kaplan, "Joining Processes an Introduction,” John Wiley and Sons, New York, 1997.

[4] S. J. Hu., G. E. Lim and T. L. Lim, "Study of Temperature Parameter on the Thermosonic Gold Wire Bonding of High-Speed CMOS,” IEEE Journal of Transactions on Components, Hybrids and Manufacturing, Vol. 14, No. 4, 1991, pp. 855-858. doi:10.1109/33.105145

[5] Y. X. Wu, Z. L. Long, H. Lei and Z. Jue, “Temperature Effect in Thermosonic Wire Bonding,” Journal of Transactions on Non-ferrous Materials Society, Vol. 16, No. 3, 2006, pp. 618-622. doi:10.1016/S1003-6326(06)60109-X

[6] Z. L. Long, H. Lei, Y. X. Wu and Z. Jue, "Study of Temperature Parameter in Au-Ag Wire Bonding," IEEE Journal of Transactions on Electronics Packaging Manufacturing, Vol. 31, No. 3, 2008, pp. 221-226. doi:10.1109/TEPM.2008.926278

[7] H. Lei, F. L. Wang and W. H. Xu, "Bondability Window and Power Input for Wire Bonding," Journal of Microelectronics Reliability, Vol. 46, No. 2-4,2008, pp. 610-615. doi:10.1016/j.microrel.2005.05.018

[8] N. Srikanth, S. Murali and Y. M. Wong, "Critical Study of Thermosonic Copper Ball Bonding," Thin Solid Flims, Vol. 462-463, 2004, pp.339-345. doi:10.1016/j.tsf.2004.05.116

[9] M. Y. Li, H. J. Ji and C. Wang, "Interdiffusion of Al-Ni System Enhance by Ultrasonic Vibration at Ambient Temperature,” Journal of Ultrasonics, Vol. 45, No. 1-4, 2006, pp. 61-65 doi:10.1016/j.ultras.2006.06.058

[10] D. S. Liu and C. Y. Ni, "A Thermo Mechanical Study on the Electrical Resistance of Aluminum Wire Conductors," Microelectonics Reliability, Vol. 42, No. 3, 2002, pp. 367374. doi:10.1016/S0026-2714(01)00258-X

[11] S. Murali, "Formation and Growth of Intermetallics in Thermionic Wire Bonds: Significance of Vacancy-Solute Binding Energy," Alloys Compounds, Vol. 426, No. 1-2, 2006, pp. 200-204. doi:10.1016/j.jallcom.2006.08.052

[12] L. Cohen, “Time-Frequency Analysis,” Prentice-Hall, Eaglewood Cliffs, New Jersey, 1995.

[13] D. Zhang and S. Ling, "Monitoring Wire Bonding via Time-frequency Analysis of Horn Vibration,” IEEE Transactions Electronics Packaging Manufacturing, Vol. 26, No. 3, 2006, pp. 216-220. doi:10.1109/TEPM.2003.820800 